\title{
Tendencias de mortalidad en población adulta, Medellín, 1994-2003
}

\author{
Doris Cardona, Héctor Byron Agudelo \\ Grupo de Investigación Demografía y Salud, Universidad de Antioquia, Medellín, Colombia
}

Introducción. La muerte de un adulto afecta tanto su familia como a su entorno laboral y social; en los países en desarrollo la carga de enfermedades y traumatismos sufridos por los adultos va en aumento y exige la intervención inmediata de las autoridades de salud pública.

Objetivo. Determinar la tendencia de la mortalidad según el sexo, de los adultos de 20 a 64 años de la ciudad de Medellín, en la década 1994-2003.

Materiales y métodos. Es un estudio descriptivo longitudinal retrospectivo con análisis de tendencia de las tasas brutas de mortalidad; la fuente de información secundaria fueron los certificados de defunción registrados por el Departamento Administrativo Nacional de Estadísticas en la década 1994-2003.

Resultados. La tasa promedio de mortalidad de la década fue de 497 fallecimientos por cien mil habitantes; los hombres presentaron un riesgo de morir 3,5 veces el riesgo de las mujeres (IC95\%: $2,05-5,96$ ) y $58,7 \%$ de las muertes tuvieron origen en una causa externa; $28,9 \%$ de las defunciones femeninas se originaron por tumores o neoplasias y su tendencia es positiva a lo largo de los años. Las cinco primeras causas de muerte, son: agresiones, enfermedad isquémica del corazón, accidentes de transporte, tumores malignos y enfermedad pulmonar.

Conclusiones. La mortalidad de la población adulta presenta diferencias según sexo y edad, estando en condiciones más desventajosas la población masculina frente a la femenina, de fallecer por causas externas en edades más tempranas de la vida, y los de mayor edad principalmente las mujeres, por el incremento de las enfermedades cardiovasculares y los tumores malignos, que representan un problema de salud pública por el alto costo humano y económico que generan.

Palabras clave: tasa de mortalidad/estadística y datos numéricos, adulto, esperanza de vida/ tendencias, años potenciales de vida perdidos, causa de muerte/tendencias.

Trends in adult mortality in the city of Medellín, Colombia, 1994-2003

Introduction. The death of an adult affects the family as well as the adult's role in its labor and social environment. In developing countries, the load of diseases and associated trauma continues to increase and place increasing demands for the intervention by public health authorities.

Objective. Trends of mortality were determined for adults of 20 to 64 years in Medellín, Colombia, between 1994 and 2003 according to sex.

Materials and methods. A retrospective, longitudinal study was undertaken that analyzed trends in mortality rates. A secondary data source consisted of death certificates recorded at the Departamento Administrativo Nacional de Estadísticas (National Department of Statistics) during the decade 1994-2003.

Results. The rate average of mortality for the decade was 497 deaths per 1000 habitants. Men displayed a risk of dying of $3.5(95 \% \mathrm{Cl}: 2.0-6.0)$ times that of women. Approximately $59 \%$ of the deaths had external causes (i.e., not related to illness or old age). Twenty-nine percent of the female deaths were due to tumors; this trend was positive throughout the decade. The 5 most important causes of mortality were as follows: aggressive behaviors, heart disease, traffic accidents, malignant tumors and pulmonary disease. The trend in deaths due to by external causes was negative, but positive for the deaths due to acute myocardial infarctions (mainly in the 45 to 64 year population) and HIV in young adults. An increasing trend in malignant breast 
cancer was observed in young adult women, but remained constant in the 45 to 64 year-old female population.

Conclusion. Mortality in the adult population differs by sex and age. The younger male population is more at risk than women as a consequence of environmental factors in early life. In later life, women are more strongly affected by increasing incidence of cardiovascular disease and malignant tumors. The increased levels of mortality at all age levels represent a problem that generates a high human and economic cost.

Key words: Mortality rate/statistics \& numerical data, adult, life expectancy/trends, potential years of life lost, cause of death/trends.

La edad adulta es la etapa del ciclo de vida con mayor exposición y acumulación de factores de riesgo, que lo hacen proclive a desarrollar más tempranamente enfermedades crónicas, no transmisibles y mentales; sumado a otros factores adversos, aumenta la mortalidad por causas externas, como accidentes de tránsito y lesiones, además de los pocos controles médicos que se realizan, propician una tendencia a la automedicación, subvaloración del riesgo y letalidad con consecuencias funestas (1).

La probabilidad de morir de un individuo es muy diferente al tipo de enfermedad padecido; hay patologías de alta incidencia pero raramente son causas de muerte (2). Las estadísticas de morbilidad permiten conocer de qué se enferman o qué padecen los habitantes de determinada región, calcular la población por atender con determinados programas y la demanda de servicios que esa atención exigirá (3). A pesar de los notables avances registrados por los sistemas de información en salud del país, aún existen grandes deficiencias en la información, en especial sobre morbilidad por carencia de datos, subregistro y falta de compromiso real de los actores involucrados en el desarrollo e implementación de los sistemas de información en forma efectiva y eficaz $(2,4)$.

La asociación entre edad y estado de salud de las personas hace posible predecir el desarrollo de ciertas enfermedades, si se conoce la proporción que representan los diferentes grupos de edad en la población. Generalmente, los grupos

\footnotetext{
Correspondencia:

Doris Cardona Arango, Facultad Nacional de Salud Pública, Universidad de Antioquia, calle 62 No. 52-59.

Teléfono: 210 6867; fax: 5112506

doriscar@guajiros.udea.edu.co
}

Recibido: 21/11/06; aceptado: 02/05/07 de edad más estudiados en cuanto a morbilidad desviación objetiva o subjetiva de un estado de bienestar fisiológico-son los menores de un año y los mayores de 65, quienes más acceden a los servicios de salud debido a sus condiciones físicas; no obstante, la población adulta presenta un perfil epidemiológico relacionados con la actividad laboral, mano de obra productiva y población económicamente activa.

Los traumatismos accidentales e intencionales afectan principalmente a los adultos jóvenes y tienen a menudo graves consecuencias que causan discapacidad. Los accidentes de tráfico se constituyen en la tercera causa de morbilidad a nivel mundial, antecedida por el $\mathrm{VIH} /$ sida y el trastorno depresivo mayor; las autolesiones, el suicidio y los actos de violencia y de guerra representan una parte cada vez mayor de la carga, sobre todo en adultos jóvenes económicamente productivos. En los países desarrollados, los suicidios acaparan la proporción más importante de la carga atribuible a los traumatismos intencionales, mientras que en las regiones en desarrollo es la violencia y la guerra las que ocupan el primer lugar (5).

La muerte de un adulto afecta a su familia, su entorno laboral y social; los esfuerzos internacionales encaminados a comprender la magnitud de los retos sanitarios que encaran los países en desarrollo todavía son incipientes, incluso impera la idea de que la salud de los adultos sólo constituye una preocupación importante en los países ricos, donde se ha logrado reducir notablemente la mortalidad prematura entre los niños. Sin embargo, la carga de enfermedades y traumatismos sufridos por los adultos en los países en desarrollo va en aumento y exige la intervención inmediata de las autoridades de salud pública de todo el mundo (5). 
La mortalidad -acción de la muerte sobre una población- es un indicador indirecto de las condiciones de salud; sus niveles, tendencias, diferencias y causas constituyen elementos básicos que deben considerarse en la planificación de los servicios de salud (6). La mortalidad es un indicador de la magnitud y del riesgo absoluto de morir que refleja las condiciones de vida de la población, la utilización de los servicios de salud, los avances tecnológicos, los niveles de educación, el urbanismo planificado, la prestación de los servicios de salud, el desarrollo de la región, el quehacer de los diferentes sectores y las enfermedades que padece la población y por las cuales está muriendo (7).

El informe sobre la salud en el mundo del año 2003 (5) cataloga a Colombia en los países en desarrollo con baja mortalidad, con una mayor proporción de la población de 60 y más años, seguido de los adultos entre 15 y 59 años, y, por último, la población menor de cinco años; se resalta el elevado número de muertes en adultos de 15 a 59 años en los países en desarrollo, con más de $30 \%$ del total de fallecimientos, mientras en los países desarrollados se sitúa en $20 \%$.

Los eventos conocidos como de causalidad externa relacionados con la violencia y los accidentes de tránsito han registrado un incremento notable, y se han constituido en uno de los principales problemas de salud pública de la actualidad (7). Entre todos los países de América, Colombia tiene uno de los más altos índices de violencia; se estima que $15 \%$ se debe a causas políticas y $85 \%$ a conflictos cotidianos (7).

En Medellín, la evolución de la estructura de la mortalidad evidencia tanto problemas característicos de las condiciones de vida como de los conflictos propios de los países subdesarrollados. Hasta el periodo de 1947-1951, predominaron las enfermedades infecciosas y respiratorias como las primeras causas de mortalidad; posteriormente, de 1962-1985, se incrementaron las afecciones cardiovasculares y, aunque los accidentes de tránsito, envenenamientos y violencia ocuparon el tercer lugar en la mortalidad desde 1972, a partir de 1986 se ubican en el primer lugar (6).
Al hacer la búsqueda de la información existente sobre morbilidad y mortalidad para los adultos de Medellín, se observó una carencia de información con respecto a la población adulta joven (de 20 a 44 años de edad), ya que actualmente se hace una agrupación de la población de 15 a 44 años de edad, sin considerar que, debido a las condiciones sociales y económicas, se presentan diferentes características en su situación de salud $(6,8)$.

Como un componente del proyecto de investigación "Aspectos de la calidad de vida de la población adulta: comparativo por sexo. Medellín, 2005" (9), se analizó la tendencia de la mortalidad de los adultos de 20 a 64 años de Medellín, con el fin de determinar la pérdida de años productivos de la ciudad en la década 19942003.

\section{Materiales y métodos}

Este es un estudio descriptivo longitudinal retrospectivo con análisis de tendencia de las tasas brutas de mortalidad entre 1994-2003, comparativo entre hombres y mujeres de 20 a 64 años fallecidos en Medellín en el período de estudio; la fuente de información secundaria fueron 56.541 certificados de defunción registrados en la base de datos del Departamento Administrativo Nacional de Estadísticas (DANE) (10).

Se incluyeron registros de hombres y mujeres fallecidos en los años seleccionados cuya residencia habitual hubiese sido Medellín; se excluyeron los registros que no tenían definido el sexo, la edad y el lugar de residencia.

El plan de análisis fue univariado por sexo, edad y causa básica de la muerte, y bivariado en la comparación de tasas de mortalidad específica por sexo y grupos de edad; se utilizó el método epidemiológico de estandarización o ajuste de tasas, con el fin de remover el efecto confusor de variables que se sabe -o supone- difieren entre hombres y mujeres (11). Estas tasas se ajustaron por el método directo por edad para cada sexo, a partir de los 15 hasta 65 y más años de edad, y se calcularon utilizando como población estándar la estructura por edad de la población de la ciudad en el año 2005, con cifras oficiales del 
Departamento Administrativo de Planeación Municipal (12).

También se calcularon las tablas de vida y la esperanza de vida (13) con el método Arriaga (14) y para los años de vida potencialmente perdidos (15) se calcularon por grupos quinquenales de edad, teniendo como punto de corte la esperanza de vida al nacer (69 años para los hombres y 74 para las mujeres), utilizando el programa Epidat versión 3.1 (16). Se calcularon las tendencias de mortalidad (17) de las cinco primeras causas para cada sexo y para la agrupación de adulto joven (de 20 a 44 años) y adulto medio (de 45 a 64 años) por medio de la prueba estadística de ji al cuadrado de la tendencia.

Para la causa básica de muerte -enfermedad o lesión que inició la cadena de acontecimientos, o las circunstancias del accidente o violencia que produjo la lesión fatal- (2), se tomó la Clasificación Internacional de las Enfermedades, décima revisión (CIE-10) (17-21), con la agrupación de los seis grandes grupos de la Organización Panamericana de la Salud (Lista 6/67 OPS) (2).

\section{Resultados}

Los registros estudiados corresponden a 56.990 certificados de defunción de población de 20 a 64 años fallecida en Medellín en la década 1994-2003 y fueron excluidos $449(0,79 \%)$ por no cumplir con los criterios de inclusión; por ello, se analiza la información de 56.541 registros.

La tasa de mortalidad total en los 10 años estudiados fue de 5,0 fallecidos por cada mil habitantes adultos de la ciudad; la tasa más alta se presentó en 1994 con 6,4 y disminuyó a lo largo de los años hasta ubicarse en el 2003 en 3,6 muertes por mil habitantes adultos en el 2003. Para los adultos masculinos la mortalidad fue de 8,2 por mil habitantes; la más alta se presentó en 1994, con 11,1 defunciones por cada mil habitantes, y la más baja, en el 2003 con 5,5. Las adultas registraron una tasa de 2,4 por mil mujeres adultas de la ciudad; la más alta se dio en 1996 y 1997, con 2,7, y la más baja en 2000, 2001 y 2003, con 2,1 cada uno.

El riesgo de morir de los hombres adultos fue 3,5 veces (IC95\%: 2,05-5,96) el riesgo de las mujeres en la década estudiada. Se evidenció una tendencia estadísticamente significativa en la disminución del riesgo de morir de la población masculina $\left(\chi^{2}=1.186 ; p=0,0000\right)$ y la tendencia del riesgo de morir de la población femenina es positiva en los primero cuatros años de estudio y se disminuye en los demás ( $\left.\chi^{2}=108 ; p=0,0000\right)$.

Por grupo de edad, los hombres de 60 a 64 años presentaron la tasa más alta, con 2.031,7 por cien mil habitantes y, la más baja en la edad de 40 a 44 años, con 560,9 fallecidos por cien mil hombres adultos; en las mujeres, la tasa más alta se presentó entre los 60 y los 64 años con 1.219 fallecidas por cien mil y, la más baja, en mujeres de 20 a 24 años con 89,5 . No se presentó diferencia significativa en la tendencia de mortalidad en los hombres adultos según el grupo de edad $\left(\chi^{2}=0,40 ; p=0,527\right)$; pero, sí se presentó tendencia positiva en la tasa de mortalidad de las mujeres adultas según grupo de edad $\left(\chi^{2}=9.586\right.$; $\mathrm{p}=0,0019$ ).

Agrupación OPS. Según los seis grandes grupos, la tasa promedio de mortalidad fue de 2.348 por cada cien mil habitantes fallecidos por causas externas, 676 por neoplasias, 663 por enfermedades del sistema circulatorio, 561 por las demás causas y 212 defunciones ocasionadas por enfermedades transmisibles. Los hombres fallecieron principalmente por causas externas, con 4.836 adultos masculinos fallecidos por cien mil habitantes y, por enfermedades del sistema circulatorio, 870; las mujeres fallecieron principalmente por tumores y neoplasias con una tasa de 689 por cien mil habitantes femeninas y, de 498 , por enfermedades del sistema circulatorio.

Los hombres adultos aportaron $58,7 \%$ a los fallecimientos por causas externas y un $10,6 \%$ por enfermedades del sistema circulatorio; por su parte, las mujeres adultas tuvieron mayor participación con defunciones por neoplasias o tumores, 28,9\%; por enfermedades del sistema circulatorio, 20,9\% (3.155), y por todas las demás enfermedades, $20 \%$.

Se registró una asociación estadística entre fallecer por causa externa y pertenecer al sexo masculino, lo cual indica el riesgo al que están expuestos los hombres de morir por alguna causa 
violenta inflingida o autoinflingida de siete veces el riesgo de las mujeres (OR=7,73; IC95\%: 7,36$8,11)$, y por cada mujer adulta fallecida por causa externa se dieron 13 fallecimientos de hombres adultos por la misma causa. También existe asociación estadística entre morir a causa de un tumor y ser mujer, pues presentan un riesgo cuatro veces el de los hombres (OR=4,66; IC95\%: 4,434,96). Por enfermedades transmisibles el riesgo de morir de los hombres es de $30 \%$ frente al riesgo de las mujeres (OR=1,30; IC95\%: 1,18-1,43).

Cinco primeras causas. La población de estudio se distribuyó en dos grandes grupos: adulto joven (20 a 24 años) y adulto medio (45 a 64 años), por considerar que las causas de muerte varían con la edad entre el mismo sexo; por ello, se controló la mortalidad por edad como posible variable de confusión.
Durante los años 1994 a 2003, las cinco primeras causas ocasionaron más de $70 \%$ de los fallecimientos de hombres adultos jóvenes, principalmente por causas externas, como agresión con arma de fuego de todo tipo, con una tasa promedio de 463,6 por cien mil habitantes hombres de 20 a 44 años residentes en la ciudad; la agresión con objeto cortante o punzante registró una tasa de 43,8 y los accidentes de transporte de vehículo automotor, tanto como pasajero como peatón, presentaron una tasa de mortalidad de 34; por causas infecciosas, como virus de inmunodeficiencia humana (VIH), fallecieron 15 jóvenes de sexo masculino y 12 por infarto agudo del miocardio (figura 1).

Del total de causas de defunción en la población masculina de adulto medio, las cinco primeras representan más de $35 \%$ del total de causas. Se

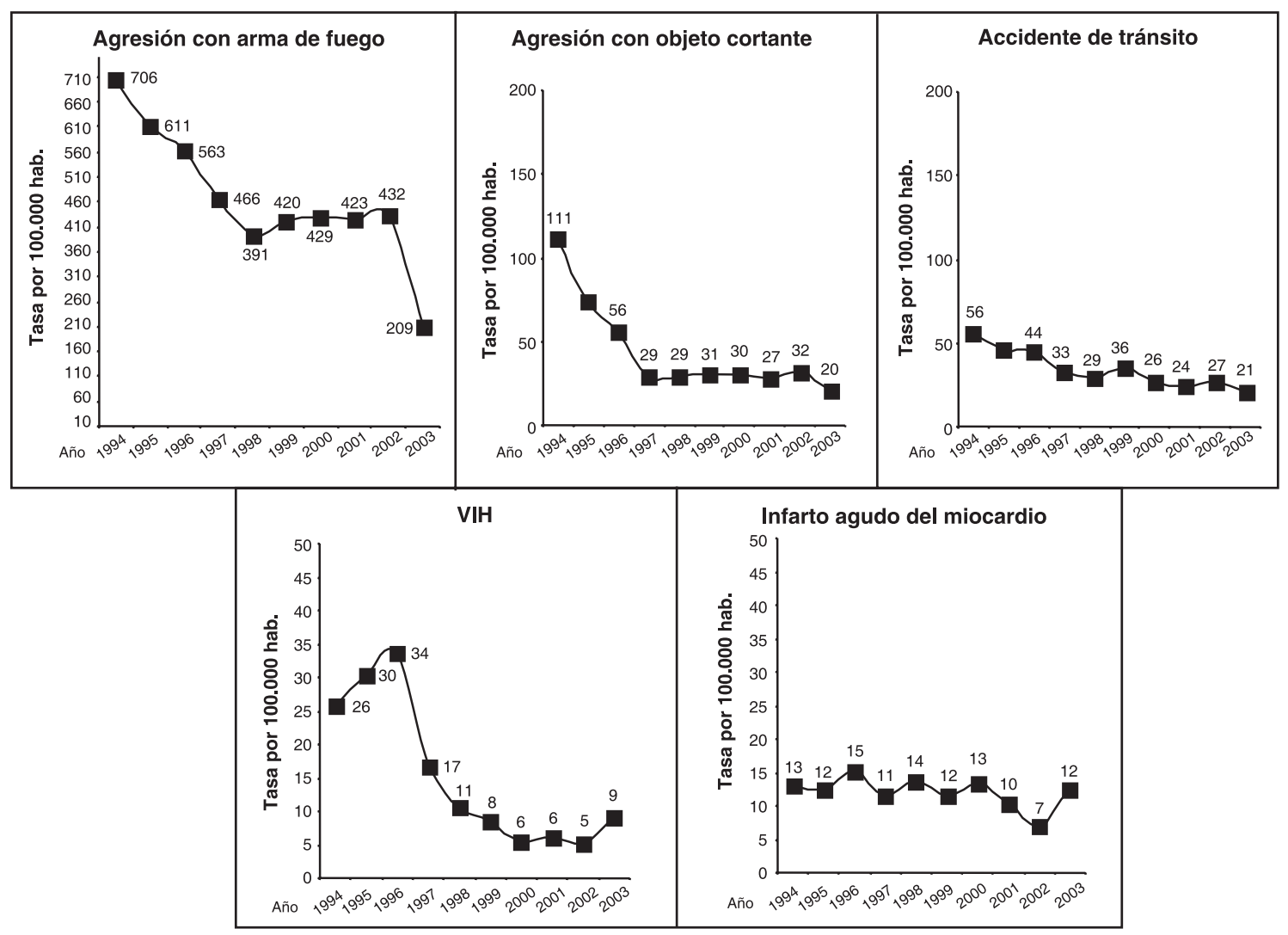

Figura 1. Tendencia de las cinco primeras causas de mortalidad en la población masculina de 20 a 44 años de edad, Medellín, 1994-2003 
encontró que la primera causa, infarto agudo del miocardio, ocasionó una tasa promedio de mortalidad de 207,6 por cada cien mil habitantes; la agresión con arma de fuego ocasionó una mortalidad de 149 defunciones por cien mil habitantes; el tumor maligno de los bronquios o del pulmón fue la tercera causa que más muertes ocasionó en la población masculina con una tasa promedio de 55, la enfermedad pulmonar obstructiva crónica ocasionó 43 defunciones por cien mil hombres y el tumor maligno del estómago presentó una tasa de 41 (figura 2).

Las cinco primeras causas de muerte de la población femenina adulta joven representan entre $50 \%$ y $30 \%$ del total de causas. A lo largo de los años se pudo observar que la principal causa de muerte fue la agresión con arma de fuego, con una tasa promedio de mortalidad de 21,8 por cien mil habitantes femeninas de este mismo grupo de edad, seguida del fallecimiento por infarto agudo del miocardio con 4,7; luego, se encontró el tumor maligno del cuello del útero con 4,3 defunciones, el tumor maligno de la mama con 4,3 y los accidentes de transporte con una tasa de mortalidad de 4,0 (figura 3).

Para la población femenina adulta media, las cinco primeras causas de defunción representaron más de $25 \%$ del total de causas para ese año. La tasa de mortalidad promedio del infarto agudo del miocardio fue de 71,4 muertes en población adulta femenina por cada cien mil habitantes de este sexo y en ese mismo rango de edad; el tumor maligno de mama aportó 32,8 de las muertes; la enfermedad pulmonar obstructiva fue la tercera
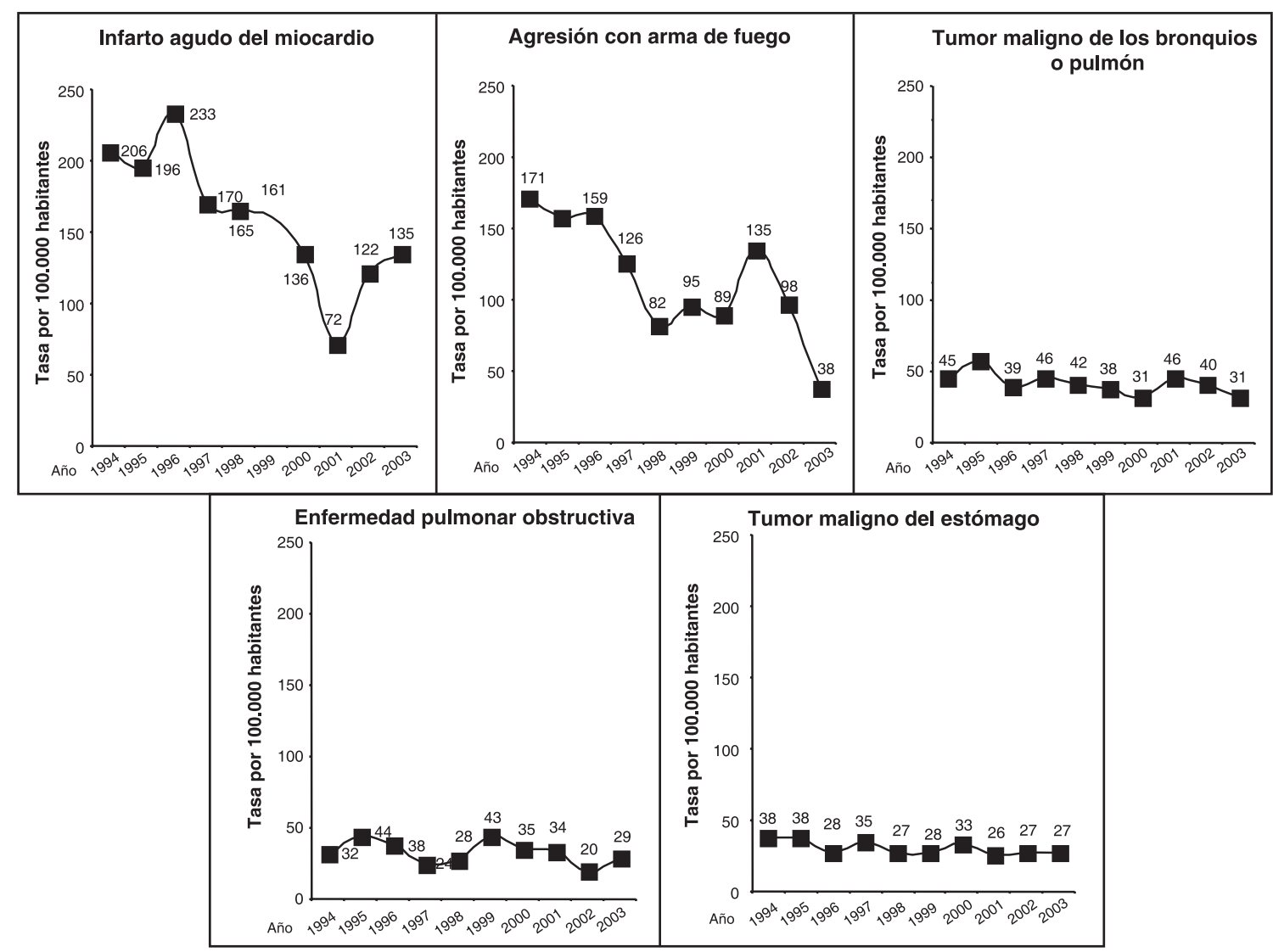

Figura 2. Tendencia de las cinco primeras causas de mortalidad en la población masculina de 45 a 64 años de edad, Medellín, 1994-2003. 


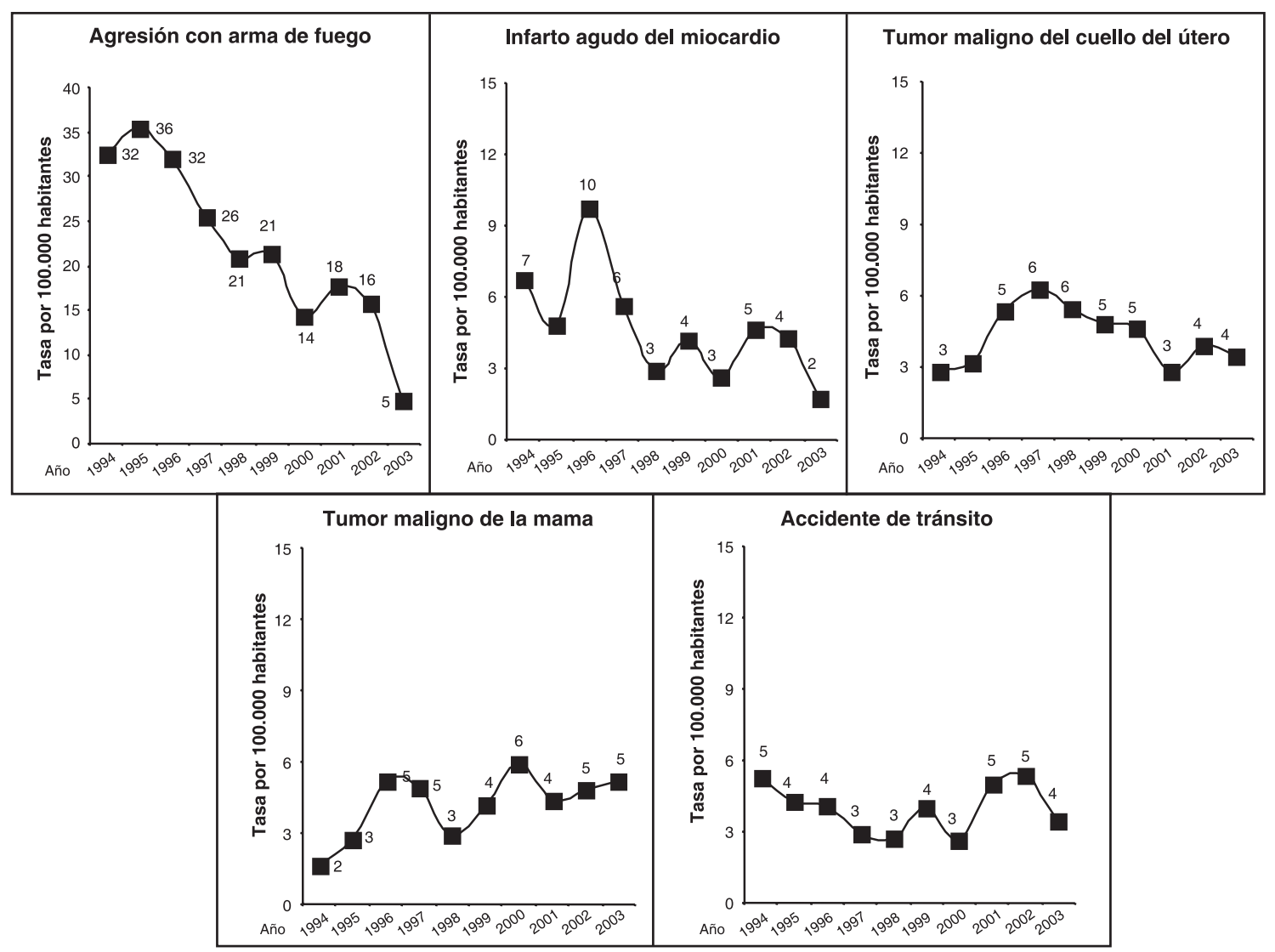

Figura 3. Tendencia de las cinco primeras causas de mortalidad en la población femenina de 20 a 44 años de edad, Medellín, 1994-2003

causa con una tasa de 32; el tumor maligno de los bronquios o del pulmón y el tumor maligno del cuello del útero ocasionaron 27 fallecimientos por cien mil habitantes femeninas, respectivamente. Se destaca el hecho que éste es el único grupo de población donde las cinco primeras causas de muerte fueron causas naturales (figura 4).

Esperanza de vida. La esperanza de vida de la población adulta de la ciudad se derivó del cálculo de las tablas de vida para cada año de estudio. La información mostró cómo se han ganado años en esperanza de vida a lo largo del período de estudio, pero con mayor ganancia en unos grupos de edad y en un sexo específico, como fue el caso de la población de 20 a 24 años que ganó cuatro años de 1994 a 2003 al pasar de 51 a 55 años; los hombres ganaron siete años al pasar de 43 a 50 años, mientras las mujeres sólo ganaron un año pasando de 58 a 59 años en este mismo grupo de edad. Los de 25 a 29 años ganaron dos años al pasar de 48 a 50; los hombres lograron cuatro años mientras la mujer se mantuvo en los 54 años de esperanza de vida. Así, se van teniendo ganancias cada vez menores en la esperanza de vida general; para las mujeres de 25 años en adelante no hay muchos años ganados porque los cambios que se dieron favorecieron a aumentar la supervivencia de los hombres, que en el grupo etario de 20 a 24 años la probabilidad de morir en el año 1994 era de 8\% y para el 2003 bajó a 3\%.

A partir del grupo de edad de 45 años en adelante, no se registra ganancia en la esperanza de vida de la población adulta de la ciudad; mientras para los hombres se mantiene estable la esperanza de vida, la de la mujer decrece a partir de los 50 


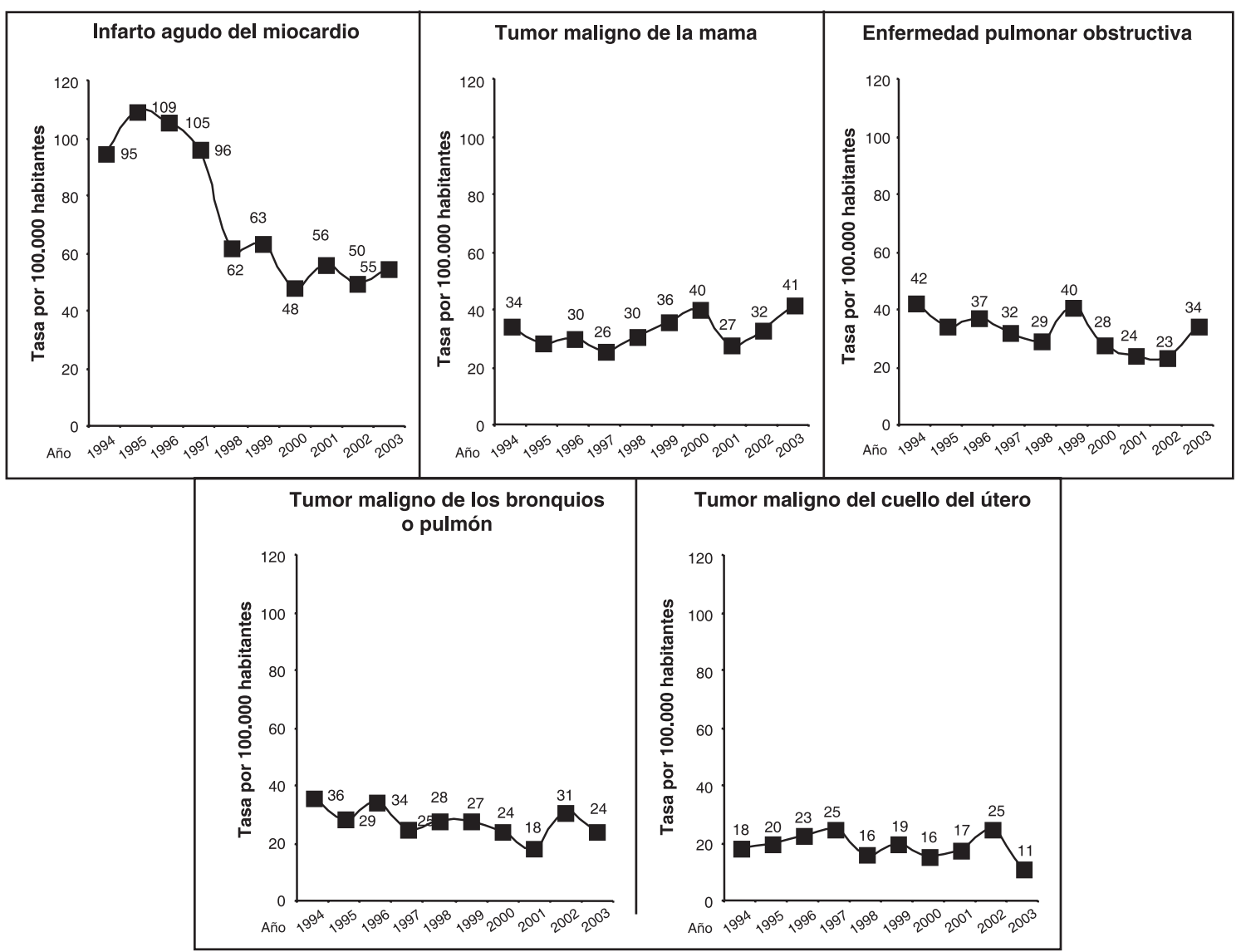

Figura 4. Tendencia de las cinco primeras causas de mortalidad en la población femenina de 45 a 64 años de edad, Medellín, 1994-2003.

años de edad y pierde un año cuando llega al grupo de de 60 a 64 años.

Años de vida potencialmente perdidos. Durante los 10 años de estudio, los hombres adultos entre 15 y 65 años aportaron más años de vida potencial que las mujeres en esta misma edad; mientras la población femenina aportó 34.193 años en 1994, la población masculina aportó 3,6 veces esa cantidad en ese mismo año y así sucesivamente, los hombres hacen un aporte mayor a los años perdidos en su etapa productiva. En la década de estudio, el mayor aporte en años perdidos de los hombres económicamente activos fue en 1996, en una relación 3,7:1, es decir, por cada año aportado por una mujer fallecida se aportaron 3,7 años de un hombre fallecido en esta misma etapa; el año de menor aporte masculino fue el 2003, con una relación de 2,6:1.
La tasa ajustada por edad mostró que por cada mil habitantes de la ciudad en 1994, se perdieron 97 años por mil personas adultas en la etapa económicamente activa; en 1995, se perdieron 85 y, así, se va disminuyendo la pérdida hasta 2003 donde sólo se pierden 42 años por cada mil personas en edad productiva o potencialmente productiva. En la pérdida de años de 1994, los hombres aportaron 158 años por cada mil habitantes masculinos, mientras las mujeres perdieron 44 años por cada mil mujeres potencialmente activas de la ciudad; en 2003, la tasa de los hombres fue de 63 años y de las mujeres 23 años, por cada mil habitantes del mismo sexo.

No se observó una tendencia estadísticamente significativa en el comportamiento de los años de vida potencialmente perdidos por la población 
masculina $\left(\chi^{2}=0,454 ; p=0,501\right)$, tampoco en la población femenina $\left(\chi^{2}=0,269 ; p=0,6038\right)$, ni en la población total económicamente activa entre 15 y 65 años de edad de la ciudad de Medellín $\left(\chi^{2}=0,401 ; p=0,5263\right)$.

\section{Discusión}

Antes de analizar la mortalidad de la población adulta en la década 1994-2003 registrada en el DANE (10) y Medellín, se deben recordar las limitaciones existentes por el subregistro que presentan las bases de datos; aunque fueron suministradas por entidades oficiales, éstas pueden estar afectadas en cualquier etapa de su producción, como recolección de datos, codificación o procesamiento del dato; y pueden originarse en las limitaciones de conocimientos médicos, como errores de diagnóstico, deficiencias en la certificación y una comprensión insuficiente de los usos que se hacen de la información proporcionada en ellos $(2,13)$.

Para Colombia, el subregistro estimado en los certificados de defunción en el año 2002 fue de $25 \%(4,13)$ y, entre $2000-2005$, de $17,5 \%$ (22), lo que afecta la posibilidad de comparar los datos con otros países y otras regiones del país. La fuente de información secundaria que recopiló los datos primarios de personas adultas fallecidas en la década estudiada, registró $1 \%$ en causas de defunción del grupo 7 correspondiente a "signos y síntomas mal definidos" de la lista OPS 6/67, $10 \%$ de registros incompletos y $0,79 \%$ sin diligenciamiento en las variables edad o sexo del fallecido, que pudiera estar afectando la información sobre mortalidad de los adultos de la ciudad, al sobrepasar los estándares internacionales definidos por la OPS, que estipula que las categorías residuales y las mal definidas no deben sobrepasar $10 \%(2,13)$ y, en este caso, fue de $12 \%$.

Las causas de defunción difieren por sexo; los hombres de 20 a 44 años aportaron más a las causas externas, con muertes ocasionadas por arma de fuego, arma cortopunzante y accidentes de transporte. Para las mujeres del grupo adulto joven, las causas de fallecimiento estuvieron relacionadas con causas externas por agresión con arma de fuego, seguida de fallecimiento por infarto agudo del miocardio, tumor maligno del cuello del útero, tumor maligno de la mama y accidentes de transporte.

Este comportamiento está acorde con los hallazgos de la Secretaría de Salud Municipal (23), en los que la mortalidad por agresión es la principal causa de muerte, con tasas de incidencia que fluctúan entre 264 y 253 por diez mil habitantes entre 1992 y 2001, pero, muy superiores a las registradas en Colombia entre 2000 y 2005, con una tasa ajustada de 183,4 por cien mil habitantes masculinos y 38,4 en población femenina (22), acorde con el Informe de la Salud en el Mundo 2003 (5), en el cual los traumatismos por accidente de tráfico fueron la tercera causa de mortalidad en el mundo en la población de 15 a 59 años, y las autolesiones y la violencia, la sexta y la séptima causas, respectivamente.

La asociación estadística es una evidencia de la relación existente entre fallecer por causa externa y pertenecer al sexo masculino, es un indicativo del riesgo de morir por causa violenta inflingida o autoinflingida al que están expuestos los hombres adultos, superior en siete veces al riesgo de morir de las mujeres por esta misma causa. La mortalidad por causas externas hace de Medellín uno de los municipios con mayores tasas de muerte en el país hasta el 2003 (23), comparándola con otras ciudades como Bogotá y Cali, que presentaban tasas de 70 y 80 por cien mil habitantes, con Colombia, de 69, y con América Latina donde la tasa es de 19 por cien mil. Sólo el caso de Medellín es comparable a países como El Salvador, que ha visto aumentar sus niveles de violencia después de las negociaciones de paz en los años 90 (24).

El fallecimiento por VIH presentó una tasa de mortalidad de 15 por cien mil habitantes adultos jóvenes (de 20 a 44 años) de sexo masculino de la ciudad, pero es a partir de 1997 cuando se posicionó entre las primeras causas según laSituación de Salud de Medellín (23), con tasas que oscilan entre 13,7 y 11,8 ; esto corrobora lo hallado en la población de 15 a 59 del mundo, en la cual la primera causa de muerte en el 2002 fue el $\mathrm{VIH} /$ sida con 2.279 defunciones (5). Esta causa también cobró vidas en el sexo femenino, pero no se ubicó entre las primeras cinco. 
Para la población adulta media (de 45 a 64 años), durante los años 1994-2003, una de las principales causas de muerte fue el infarto agudo del miocardio, que afecta tanto a hombres como mujeres, confirmando así lo expuesto por otros estudios que plantea cómo las enfermedades crónicas debidas a problemas respiratorios y tumores malignos han aportado un importante número de muertes (25). El infarto agudo del miocardio es la segunda causa en el mundo después del VIH y es la que con el paso del tiempo generará mayor número de muertes (5).

El incremento gradual de las enfermedades cardiovasculares, tales como problemas hipertensivos, cardiopatía coronaria, enfermedad isquémica del corazón, enfermedades cerebrovasculares y otras que afectan el corazón, es una importante causa de mortalidad y representa un problema de salud pública por el alto costo humano y económico que generan; son, además, la causa principal de muerte en la población mayor de 45 años (7), causa común con los países industrializados (24), en Colombia, la enfermedad isquémica del corazón se ha presentado como la principal causa de muerte por enfermedades del sistema circulatorio (23).

En conclusión, no se evidenció tendencia en la tasa de mortalidad en los hombres adultos de la ciudad según el grupo de edad, pero las mujeres adultas sí presentaron tendencia al incremento. Según la mortalidad por las cinco primeras causas, en este estudio se observó tendencia negativa en la mortalidad de los hombres de 20 a 44 años en cuatro de ellas, principalmente las ocasionadas por causas externas, mientras se incrementaron las muertes por infarto agudo del miocardio en los últimos años del estudio que se conservan en la población de 45 a 64 años y aumento de fallecimientos por $\mathrm{VIH} /$ sida en población adulta joven.

En las mujeres de 20 a 44 años la tendencia es a la disminución en cuatro de las cinco causas, con excepción del tumor maligno de la mama que muestra tendencia al aumento; este ascenso se conserva en las mujeres de 45 a 64 años. El infarto agudo del miocardio es la segunda causa en mujeres adultas jóvenes y la primera causa en la adulta medio; en este grupo de población de mujeres de 45 a 64 años se presenta un incremento de fallecimientos por enfermedad pulmonar obstructiva y, como quinta causa pero con tendencia a disminuir, se encuentra el tumor maligno del cuello del útero.

A medida que se aumenta la edad se aumenta el riesgo de morir, conclusión lógica derivada del ciclo de vida natural. Pero esta lógica no se aplica en el sexo masculino, donde el riesgo de morir es alto en la etapa del adulto joven, se disminuye hasta los 35 años y a partir de los 45 inicia nuevamente el ascenso hasta los 64 años analizados.

La ganancia en años de esperanza de vida es mayor en los hombres que en las mujeres, sobre todo en los años del adulto joven; a partir de la etapa del adulto medio no se registra ganancia alguna y se conserva estable hasta terminar el período; mientras la esperanza de vida de las mujeres decrece a partir de los 50 años de edad y pierde un año cuando llega al grupo de 60 a 64 años.

Durante los 10 años de estudio, los hombres mayores de 15 años aportaron más años de vida potencialmente perdidos que las mujeres, en una relación de 3,6:1 en 1994 a 2,6:1 en el año 2003; es decir, por cada año aportado por una mujer fallecida en esta etapa productiva se aportaron 3,6 años de un hombre fallecido en esta misma etapa de la vida, perdiéndose así 97 años por mil personas adultas en la etapa económicamente activa en 1994. En 1995 se perdieron 85 y así va disminuyéndose la pérdida hasta el 2003 cuando sólo se pierden 42 años por cada mil personas en edad productiva o potencialmente productiva. $\mathrm{Si}$ el objetivo principal del trabajo de salud pública es aumentar la esperanza de vida de las poblaciones en las mejores condiciones de salud posibles (26), partiendo del conocimiento de la mortalidad en los diferentes grupos de edad, se conocen los años de vida potencialmente perdidos, los cuales ilustran la pérdida que sufre la sociedad como consecuencia de la muerte de personas jóvenes (15).

Respecto a la mortalidad de la población adulta de 20 a 64 años, se observan diferencias entre 
hombres y mujeres; la población masculina está en condiciones más desventajosas, con una tasa de mortalidad mayor que la de las mujeres, principalmente por causas externas en edades más tempranas de la vida.

\section{Agradecimientos}

A la Universidad de Antioquia, Grupo de Investigación Demografía y Salud y a la Facultad Nacional de Salud Pública, por apoyar la realización del proyecto. A las entidades oficiales: Alcaldía de Medellín a través del Departamento Administrativo de Planeación, Secretaría de Salud y al Departamento Administrativo Nacional de Estadísticas por la información secundaria suministrada.

\section{Conflicto de intereses}

Los investigadores declaramos que durante la realización de la investigación no existió conflicto de intereses entre la entidad financiadora (Universidad de Antioquia), la Secretaría de Salud Municipal y el Departamento Administrativo Nacional de Estadísticas que pudieran haber afectado los resultados del mismo.

\section{Financiación}

Comité para el Desarrollo de la Investigación, Universidad de Antioquia y Grupo de Investigación Demografía y Salud.

\section{Referencias}

1. Naciones Unidas. Informe sobre la juventud mundial 2005. Informe del Secretario General. Nueva York: Naciones Unidas; 2005.

2. Organización Panamericana de la Salud. De datos básicos a índices compuestos: una revisión del análisis de mortalidad. Bol Epidemiol. 2002;23:1-6.

3. López-Moreno S, Corcho-Berdugo A, LópezCervantes M. La hipótesis de la comprensión de la morbilidad: un ejemplo de desarrollo teórico en epidemiología. Salud Pública Mex. 1998;40:442-9.

4. Mathers CD, Fat DM, Inoue I, Rao C, López AD. Counting the dead and what they died from: an assessment of the global status of cause of death data. Bull World Health Organ. 2005;83:171-7.

5. Organización Mundial de la Salud. Informe sobre la salud en el mundo 2003. Ginebra: OMS; 2003.

6. Empresa Social del Estado Metrosalud. Diagnóstico de la situación de salud. Medellín. 1984-1993. Medellín: Metrosalud; 1994.
7. Dirección Seccional de Salud de Antioquia. Diagnóstico de la situación de salud de Antioquia. Rev Epidemiol Antioquia. 2000;25:1-233.

8. Empresa Social del Estado Metrosalud. Cronología de la atención en salud en Medellín 1986-1996. Medellín: Metrosalud, Municipio de Medellín; 1997.

9. Cardona D, Agudelo HB. La flor de la vida: pensemos en el adulto. Aspectos de la calidad de vida de la población adulta. Medellín, 2005. Medellín: Universidad de Antioquia; 2006.

10. Departamento Administrativo Nacional de Estadísticas. Certificados de defunción, Medellín 1994-2003. Bogotá: DANE; 2005.

11. Organización Panamericana de la Salud. La estandarización: un método epidemiológico clásico para la comparación de tasas. Bol Epidemiol. 2002;23:9-12.

12. Departamento Administrativo de Planeación Municipal. Proyección de Población. Medellín: Alcaldía de Medellín; 2005.

13. Organización Panamericana de la Salud. Sobre la estimación de tasas de mortalidad para países de la Región de las Américas. Bol Epidemiol. 2003;24:1-16.

14. Arriaga EE. El análisis de la población con microcomputadoras. [Tesis de doctorado en demografía]. Córdoba: Universidad Nacional de Córdoba; 2001.

15. Organización Panamericana de la Salud. Técnicas para la medición del impacto de la mortalidad: años potenciales de vida perdidos. Bol Epidemiol. 2003; 24:1-16.

16. OPS, Xunta de Galicia. EPIDAT 3.0. Programa para análisis epidemiológico de datos tabulados. La Habana: OMS; 2000.

17. Organización Panamericana de la Salud. Tendencias demográficas y de mortalidad en la Región de las Américas, 1980-2000. Bol Epidemiol. 2002;23:1-4.

18. Ministerio de Salud. Resolución 01895 de 2001, por la cual se adapta para Colombia la Clasificación Estadística Internacional de Enfermedades y problemas relacionados con la salud, CIE-10, a partir del 01 de enero de 2003. Bogotá: Ministerio de Salud; 2001.

19. Organización Mundial de la Salud. Clasificación Internacional de Enfermedades. Novena revisión. Washington D.C.: OPS; 1978.

20. Organización Mundial de la Salud. Clasificación estadística internacional de enfermedades y problemas relacionados con la salud. Décima revisión. Washington D.C.: OPS; 1995.

21. Instituto de Estadística de la Comunidad de Madrid. Correspondencia entre la CIE 10 y la CIE 9. 2004. [Consultado: 21 de diciembre de 2005.] Disponible en: 
http://www.madrid.org/iestadis/fijas/clasificaciones/ corcie.htm.

22. Organización Panamericana de la Salud, Organización Mundial de la Salud. Situación de salud en las Américas: indicadores básicos 2005. Washington D.C.: OPS/OMS; 2005.

23. Dirección Seccional de Salud. Situación de Salud de Medellín: Indicadores básicos. Medellín: Alcaldía de Medellín; 2003.
24. Zapata Y. Mortalidad en población de 10 a 19 años con énfasis en la mortalidad violenta y por lesiones. Medellín, 1998-1999 [Trabajo de grado en Tecnología de sistemas de información en salud]. Medellín: Universidad de Antioquia; 2003.

25. Dirección Seccional de Salud. Perfil de mortalidad de la ciudad de Medellín 1999-2001. Medellín: Alcaldía de Medellín; 2003.

26. Organización Panamericana de la Salud. Años potenciales de vida perdidos - Brasil, 1980. Bol Epidemiol. 1986;5:3-6. 